\title{
On the Complexity of Polynomial Matrix Computations
}

\author{
Pascal Giorgi, Claude-Pierre Jeannerod and Gilles Villard \\ CNRS, INRIA, Laboratoire LIP \\ ENSL, 46, Allée d'Italie 69364 Lyon Cedex 07, France \\ http://www.ens-lyon.fr/ $\sim$ \{pgiorgi,cpjeanne,gvillard $\}$
}

\begin{abstract}
We study the link between the complexity of polynomial matrix multiplication and the complexity of solving other basic linear algebra problems on polynomial matrices. By polynomial matrices we mean $n \times n$ matrices in $\mathrm{K}[x]$ of degree bounded by $d$, with $\mathrm{K}$ a commutative field. Under the straight-line program model we show that multiplication is reducible to the problem of computing the coefficient of degree $d$ of the determinant. Conversely, we propose algorithms for minimal approximant computation and column reduction that are based on polynomial matrix multiplication; for the determinant, the straight-line program we give also relies on matrix product over $\mathrm{K}[x]$ and provides an alternative to the determinant algorithm of $[16,17]$. We further show that all these problems can be solved in particular in $O^{\sim}\left(n^{\omega} d\right)$ operations in K. Here the "soft O" notation $O^{\sim}$ indicates some missing $\log (n d)$ factors and $\omega$ is the exponent of matrix multiplication over $\mathrm{K}$.
\end{abstract}

\section{Categories and Subject Descriptors}

F.2.1 [Analysis of Algorithms and Problem Complexity]: Numerical Algorithms and Problems-computations on matrices, computations on polynomials.

\section{General Terms}

Algorithms.

\section{Keywords}

Matrix polynomial, minimal basis, column reduced form, matrix gcd, determinant, polynomial matrix multiplication.

\section{INTRODUCTION}

The link between matrix multiplication and other basic linear algebra problems is well known under the algebraic complexity model. For $\mathrm{K}$ a commutative field, we will assume that the product of two $n \times n$ matrices over $\mathrm{K}$ can be

Permission to make digital or hard copies of all or part of this work for personal or classroom use is granted without fee provided that copies are not made or distributed for profit or commercial advantage and that copies bear this notice and the full citation on the first page. To copy otherwise, to republish, to post on servers or to redistribute to lists, requires prior specific permission and/or a fee.

ISSAC'03, August 3-6, 2003, Philadelphia, Pennsylvania, USA.

Copyright 2003 ACM 1-58113-641-2/03/0008 ...\$5.00. computed in $O\left(n^{\omega}\right)$ operations in $\mathrm{K}$. Under the model of computation trees over $\mathrm{K}$, we know that $\omega$ is also the exponent of the problems of computing the determinant, the matrix inverse, the rank, the characteristic polynomial (we refer to the survey in [5, Chap.16]) or the Frobenius normal form $[8,15]$. On an algebraic RAM, all these problems can be solved with $O^{\sim}\left(n^{\omega}\right)$ operations in $\mathrm{K}$, hence the corresponding algorithms are optimal up to logarithmic terms. Here and in the rest of the paper, for any exponent $e_{1}, O^{\sim}\left(n^{e_{1}}\right)$ denotes $O\left(n^{e_{1}} \log ^{e_{2}} n\right)$ for any exponent $e_{2}$.

Much less is known for polynomial matrices and even less for integer matrices under the bit-complexity model. Difficulties come from the size of the data (and from carry propagation in the case of integer arithmetic) which make reductions between problems hard to obtain. In this paper we investigate the case of matrices in $\mathrm{K}[x]^{n \times n}$ of degree bounded by $d$. This is motivated both by the interest in studying more concrete domains than abstract fields and by the results $[16,17]$ and $[10,22]$ : Storjohann has established an algorithm of cost $O^{\sim}\left(n^{\omega} d\right)$ for the determinant and the Smith normal form; on the other hand, for the polynomial matrix inverse, there is a straight-line program whose length is $O^{\sim}\left(n^{3} d\right)$, i.e., almost the size of the output. Besides, the latter approach gives an alternative for the determinant in $O^{\sim}\left(n^{\omega} d\right)$ (see Section 4).

These two results first ask the following question: are problems on polynomial matrices - and especially the determinant problem - harder than polynomial matrix multiplication? By slightly extending the result of Baur and Strassen [1, Corollary 5] for matrices over a field, we answer positively for the determinant, up to a constant. More precisely, we show in Section 4 that if there is a straightline program of length $D(n, d)$ over $\mathrm{K}$ which computes the coefficient of degree $d$ of the determinant, then there is a straight-line program of length no more than $8 D(n, d)$ which multiplies two $n \times n$ matrices of degree $d$.

Conversely, the second question is which polynomial matrix problems can be solved with roughly the same number of arithmetic operations as polynomial matrix multiplication. As seen above we already know that this is the case of the determinant problem [16] on an algebraic RAM using $O^{\sim}\left(n^{\omega} d\right)$ as an estimation of the complexity of matrix multiplication [6]. We will see in Section 4 that a different approach, developed independently in [10, 22], gives a straight-line program of length $O^{\sim}\left(n^{\omega} d\right)$ for the same problem.

Before studying the determinant, we shall give analogous cost estimates with RAM programs for two other problems: 
we show in Section 2 how to compute minimal bases and order $d$ matrix approximants in $O^{\sim}\left(n^{\omega} d\right)$ operations in $\mathrm{K}$; we show in Section 3 that an invertible matrix can be column reduced in time $O^{\sim}\left(n^{\omega} d\right)$ as well. Note that here column reduction is roughly lattice basis reduction for $\mathrm{K}[x]$-modules.

We further study the complexities of each of the above problems in terms of general cost functions involving polynomial matrix multiplication. To do so, we denote by $\operatorname{MM}(n, d)$ the cost of multiplying two matrices of degree $d$ in $\mathrm{K}[x]^{n \times n}$ (with $\operatorname{MM}(n)=\operatorname{MM}(n, 0)$ ) and assume without loss of generality that $n$ and $d$ are powers of two. If $\mathrm{M}(d)$ denotes the cost for multiplying two polynomials of degree $d$ in $\mathrm{K}[x]$ then we can always choose $\operatorname{MM}(n, d)=O(\operatorname{MM}(n) \mathrm{M}(d))$. For example, this gives $\operatorname{MM}(n, d)=O\left(n^{\omega} d \log d \log \log d\right)$ [6]. Using an evaluation/interpolation scheme when $\mathrm{K}$ has more than $2 d$ elements, we can take $\operatorname{MM}(n, d)=O(\operatorname{MM}(n) d+$ $\left.n^{2} \mathrm{M}(d) \log d\right)$.

As we shall see in Section 2.2, our minimal basis algorithm works recursively on the degree and leads us to define the function

$$
\mathrm{MM}^{\prime}(n, d)=\sum_{i=0}^{\log d} 2^{i} \operatorname{MM}\left(n, 2^{-i} d\right) .
$$

This will be used in Sections 2 and 3 for expressing the costs of matrix approximation and column reduction. In the same way, we use algorithms of [17] that work recursively on the dimension and whose complexity estimates rely on the function

$$
\operatorname{MM}^{\prime \prime}(n, d)=\sum_{i=0}^{\log n} 4^{i} \operatorname{MM}\left(2^{-i} n, d\right) .
$$

For instance, with $\operatorname{MM}(n, d)=\Theta\left(n^{\omega} d \log d \log \log d\right)$, both sums $\operatorname{MM}^{\prime}(n, d)$ and $\mathrm{MM}^{\prime \prime}(n, d)$ are $O^{\sim}(\operatorname{MM}(n, d))$.

We refer to the books $[11,3]$ for fundamental notions and algorithms on matrix polynomials. We may also point out to the reader that Section 2 below heavily relies on [2].

\section{MINIMAL BASIS COMPUTATION}

Many problems on matrix polynomials reduce to computing minimal approximant bases (or $\sigma$-bases) [2]. Given a matrix power series $G \in \mathrm{K}[[x]]^{m \times n}$ and an approximation order $d \in \mathbb{N}$, these bases are nonsingular $m \times m$ polynomial matrices $M$ such that

$$
M G \equiv 0 \bmod x^{d} .
$$

Minimality is made precise in Definition 2.1 below. It essentially expresses the fact that $M$ has the smallest possible row degrees.

Minimal basis computations are motivated by the following two applications, which we shall develop later in the paper: in Section 3 we will use the fact that the problem of column reducing a matrix can be solved by computing a Padé approximant and thus a minimal approximant basis; in Section 4 we further use such approximants for recovering the polynomial matrix kernels that lead to the determinant. Note that a third application is the computation of minimal matrix polynomials of linearly generated matrix sequences, as proposed in [24] and [21].

Our purpose in this section is to introduce polynomial matrix multiplication into the existing approximation algorithms. We achieve this by first adapting in Section 2.1 the $\sigma$-basis algorithm of Beckermann and Labahn [2] to exploit fast matrix multiplication over K. Roughly, the algorithm of [2] works iteratively and computes a $\sigma$-basis from a $(\sigma-n)$-basis via $n$ Gaussian elimination steps on vectors of $\mathrm{K}^{m}$. How to replace these $n$ steps on $m \times 1$ vectors with a single step on an $m \times n$ matrix was unclear. We solve this problem by resorting to the approach of Coppersmith $[7,13]$ and by using the matrix product-based $L S P$ factorization algorithm of [9]. Polynomial matrix product then arises in Section 2.2 with a divide-and-conquer version of the method of Section 2.1 that generalizes the previous studies in $[2,20]$.

This divide-and-conquer version yields the cost of $O^{\sim}\left(n^{\omega} d\right)$ which improves upon the cost of $O^{\sim}\left(n^{3} d\right)$ in [2, Theorem 6.2].

To define the type of approximants we compute, we consider as in [2] the formal power series vector

$$
f(x)=G\left(x^{n}\right)\left[1, x, \ldots, x^{n-1}\right]^{T} \in \mathrm{K}[[x]]^{m} .
$$

This allows to compress the columns of $G$ into a single one: for $1 \leq j \leq n$, the coefficients of $f$ whose corresponding power in $x$ equals $j-1$ modulo $n$ give the $j$ th column of $G$. We further call (approximation) order of $v \in \mathrm{K}[x]^{1 \times m}$ and denote by ord $v$ the integer

$$
\operatorname{ord} v=\sup \left\{\tau \in \mathbb{N}: v\left(x^{n}\right) f(x) \equiv 0 \bmod x^{\tau}\right\} .
$$

Recall also that the degree $\operatorname{deg} v$ of $v \in \mathrm{K}[x]^{1 \times m}$ is the highest degree of all the entries of $v[11, \S 6.3 .2]$. For $\sigma \in \mathbb{N}$ we then define $\sigma$-bases with respect to the rows of $G$ as follows.

Definition 2.1. A $\sigma$-basis of $G$ is a matrix polynomial $M$ in $\mathrm{K}[x]^{m \times m}$ that satisfies:

I) the rows $M^{(1, *)}, \ldots, M^{(m, *)}$ have order at least $\sigma$;

II) every $v \in \mathrm{K}[x]^{1 \times m}$ such that ord $v \geq \sigma$ admits a unique decomposition $v=\sum_{i=1}^{m} c^{(i)} M^{(i, *)}$ where, for $1 \leq i \leq$ $m, c^{(i)} \in \mathrm{K}[x]$ and $\operatorname{deg} c^{(i)}+\operatorname{deg} M^{(i, *)} \leq \operatorname{deg} v(m i n i-$ mality of the approximant).

This definition coincides with [2, Definition 3.2, p.809] when the $m$ components of the multiindex in [2] are the same. Since I) yields $M\left(x^{n}\right) G\left(x^{n}\right)\left[1, x, \ldots, x^{n-1}\right]^{T} \equiv 0 \bmod x^{\sigma}$, it suffices to take $\sigma=n d$ to get approximant $M(x)$ in (1).

For simplicity - and with no loss of generality regarding the above three applications of $\sigma$-bases - we shall restrict to the case where $\sigma$ is a multiple of $n$ and $n \leq m$.

\subsection{Via Matrix Multiplication}

To introduce matrix multiplication into $\sigma$-basis computations of [2], we use the so-called $L S P$ factorization [9] (see also [3, p. 103]): every matrix $A \in \mathrm{K}^{m \times m}$ of rank $r$ can be written as $A=L S P$ where $L \in \mathrm{K}^{m \times m}$ is lower triangular with ones on the diagonal, $S \in \mathrm{K}^{m \times m}$ has $m-r$ zero rows and $P \in \mathrm{K}^{m \times m}$ is a permutation matrix; additionally, the nonzero rows of $S$ form an $r \times m$ upper triangular matrix with nonzero diagonal entries. Let

$$
1 \leq i_{1}<i_{2}<\cdots<i_{r} \leq m
$$

be the indices of the nonzero rows of $S$. Each $i_{j}$ is then uniquely defined as the smallest index such that the first $i_{j}$ rows of $A$ have rank $j$.

In Algorithm M-Basis below, we assume we compute $L S P$ factorizations with the algorithm of [9] as described in [3, p. 103]: factors $L, S, P$ are obtained in $O(\mathrm{MM}(m))$ operations in K. Furthermore, it is not hard to verify that $L$ and 
$S$ are such that if the $i$ th row of $S$ is identically zero then the $i$ th column of $L$ is the $i$ th unit vector.

\section{Algorithm M-Basis $(G, d)$}

Input: $G \in \mathrm{K}[[x]]^{m \times n}$ with $m \geq n$ and $d \in \mathbb{N}$.

Output: a $\sigma$-basis $M \in \mathrm{K}[x]^{m \times m}$ with $\sigma=n d$.

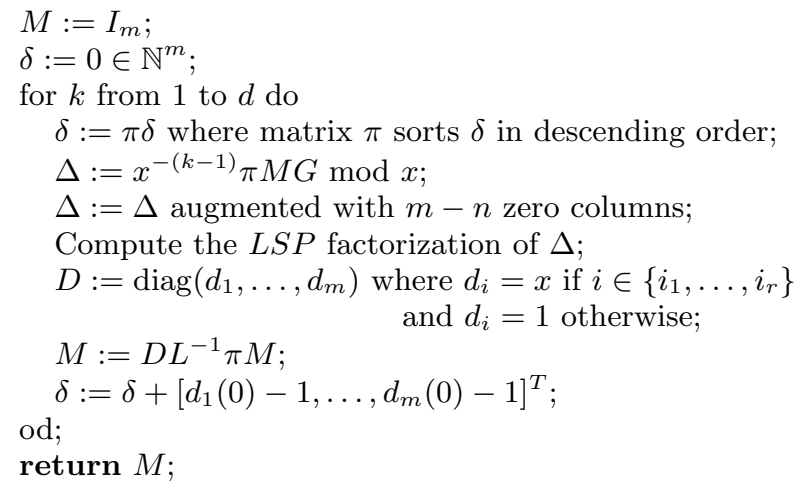

LEMMA 2.2. Algorithm M-Basis is correct. Its cost is $O\left(\mathrm{MM}(m) d^{2}\right)$ or $O\left(m^{\omega} d^{2}\right)$ operations in $\mathrm{K}$.

Proof. Let $M_{0}(x)=I_{m}$ and, for $1 \leq k \leq d$, write $M_{k}(x)$ for the matrix $M$ computed by step $k$. We see that the degree of $M_{k}$ in $x$ is no more than $k$ and, assuming the algorithm is correct, that $M_{k} G \equiv 0 \bmod x^{k}$. The computation of $\Delta$ at step $k$ thus costs $O(\mathrm{MM}(m) k)$ field operations. This dominates the cost of step $k$, for both $L S P$ factorization and the update of $M$ require only $O(\operatorname{MM}(m))$ operations in K. The overall complexity then follows.

To prove the algorithm is correct, note first that $M_{0}(x)$ is a 0 -basis of $G(x)$. Then, assuming for $k \in\{1, \ldots, m\}$ that $M_{k-1}(x)$ is an $n(k-1)$-basis of $G(x)$, we verify that $M_{k}(x)=D(x) L^{-1} \pi M_{k-1}(x)$ is an $n k$-basis of $G(x)$.

Let $N_{k-1}(x)=\pi M_{k-1}(x)$ and recall that $P$ is the permutation matrix in the $L S P$ factorization at step $k$. It follows that $N_{k-1}(x)$ is an $n(k-1)$-basis of $G(x) P^{-1}$. Algorithm FPHPS of $[2$, p. 810] with input parameters $m, n$,

$$
F(x)=N_{k-1}\left(x^{n}\right) G\left(x^{n}\right) P^{-1}\left[1, x, \ldots, x^{n-1}\right]^{T}
$$

and $(0, \ldots, 0) \in \mathbb{N}^{m}$ then returns an $n k$-basis of $G(x) P^{-1}$ after $n$ steps. We denote this basis by $N_{k}(x)$. (Uniqueness of the output of FPHPS is explained in [2, p. 818].) As shown below, the two bases are related as

$$
N_{k}(x)=D(x) L^{-1} N_{k-1}(x)
$$

and hence $M_{k}=N_{k}$ is an $n k$-basis of $G P^{-1}$ and $G$ as well.

We now prove identity (2). Let $\Lambda=\Delta P^{-1}$ and let $\Lambda_{j}$ be the $j$ th column of $\Lambda$. Then

$$
x^{-(k-1) n} F(x) \equiv \Lambda_{1}+x \Lambda_{2}+\cdots+x^{n-1} \Lambda_{n} \bmod x^{n} .
$$

Since the rows of $N_{k-1}$ have been sorted by permutation $\pi$, the first step of FPHPS simply consists in picking the first nonzero entry of $\Lambda_{1}$ - say, $\lambda_{1}$ with row index $h_{1}$ - and zeroing the lower entries of $\Lambda_{1}$ by using pivot $\lambda_{1}$. The $h_{1}$ st row is then multiplied by $x$. In other words, $N_{k-1}(x)$ is transformed into $E_{1}(x) T_{1} N_{k-1}(x)$ where we define $E_{1}(x)=$ $\operatorname{diag}\left(I_{h_{1}-1}, x, I_{m-h_{1}}\right)$ and

$$
T_{1}=\left[\begin{array}{l|l|l}
I_{h_{1}-1} & & \\
\hline & 1 & \\
\hline & t_{1} & I_{m-h_{1}}
\end{array}\right] \text { with } t_{1} \in \mathrm{K}^{m-h_{1}} .
$$

Recalling that $i_{1}$ is the index of the first nonzero row of $S$ in factorization $\Lambda=L S$, we verify first that $h_{1}=i_{1}$ : since the zero rows of $S$ correspond with unit vector columns in $L$, the product of these two matrices has the form

$$
\Lambda=L S=\left[\begin{array}{l|l|l}
I_{i_{1}-1} & & \\
\hline & 1 & \\
\hline & l_{1} & L^{\prime}
\end{array}\right]\left[\begin{array}{c|c} 
& \\
\hline \lambda_{1} & s_{1}^{T} \\
\hline & S^{\prime}
\end{array}\right], \quad \lambda_{1} \in \mathrm{K} \backslash\{0\} .
$$

Here $L^{\prime} \in \mathrm{K}^{\left(m-i_{1}\right) \times\left(m-i_{1}\right)}, S^{\prime} \in \mathrm{K}^{\left(m-i_{1}\right) \times(n-1)}$ and $\lambda_{1}$ is indeed the first nonzero entry of $\Lambda_{1}$. Hence $h_{1}=i_{1}$. Second, comparing the first column in both sides of $T_{1} \Lambda=T_{1} L S$ yields $t_{1}=-l_{1}$ and the $i_{1}$ st column of $T_{1}^{-1}$ is thus equal to the $i_{1}$ st column of $L$. The first step of FPHPS yields eventually

$$
x^{-(k-1) n} E_{1}\left(x^{n}\right) T_{1} F(x) \equiv x \Lambda_{2}^{\prime}+\cdots+x^{n-1} \Lambda_{n}^{\prime} \bmod x^{n}
$$

where

$$
\left[0\left|\Lambda_{2}^{\prime}\right| \cdots \mid \Lambda_{n}^{\prime}\right]=E_{1}(0) T_{1} \Lambda=\left[\begin{array}{c|c}
0 & 0 \\
\hline 0 & L^{\prime} S^{\prime}
\end{array}\right] \in \mathrm{K}^{m \times n} .
$$

Let $h_{2}$ be the pivot index at step 2 and let $T_{2}$ and $E_{2}(x)$ be the associated transformation matrices. It follows from (4) that $h_{2}>i_{1}$. Hence $T_{2}$ has the form $T_{2}=\operatorname{diag}\left(I_{i_{1}}, T_{2}^{\prime}\right)$ and $E_{1}(x)$ commutes with $T_{2}$. Then, noticing that the ordering imposed by $\pi$ is still the same, one can iterate by replacing $T_{1}, L S$ and $i_{1}<\cdots<i_{r}$ with respectively $T_{2}^{\prime}, L^{\prime} S^{\prime}$ and $i_{2}-i_{1}<\cdots<i_{r}-i_{1}$. We eventually get $h_{j}=i_{j}$ for $1 \leq j \leq r$. Therefore, defining for $1 \leq j \leq r$ matrices $E_{j}(x)$ and $T_{j}$ as done in (3) for $j=1$, we have

$$
N_{k}(x)=E_{r}(x) \cdots E_{2}(x) E_{1}(x) T_{r} \cdots T_{2} T_{1} N_{k-1}(x) .
$$

It follows that $E_{r}(x) \cdots E_{2}(x) E_{1}(x)=D(x)$ and that the $i_{j}$ th column of $T_{j}^{-1}$ equals the $i_{j}$ th column of $L$. Noticing further that because of the structure of $T_{j}$ the $i_{j}$ th column of $T^{-1}$ equals the $i_{j}$ th column of $T_{j}^{-1}$, we have $T^{-1}=L$ and (2) follows from (5).

\subsection{Via Polynomial Matrix Multiplication}

To use polynomial matrix multiplication, we now give a divide-and-conquer version of Algorithm M-Basis called PM-Basis. This version is based on the following "transitivity lemma", which may be seen as the counterpart of Theorem 6.1 in [2] and can be shown in the same way.

For this lemma, we need to keep track of the value of the multiindex $\delta$ involved in Algorithm M-Basis. Noting that Lemma 2.2 is actually valid for any initial value of variable $\delta \in \mathbb{N}^{m}$, one can modify this algorithm so that it takes $(G, d, \delta)$ as an input and returns $(M, \mu)$ where $\mu$ is the last value taken by $\delta$. The initialization step " $M:=I_{m} ; \delta:=0$ " thus reduces to " $M:=I_{m} ; "$.

Lemma 2.3. If $(M, \mu),\left(M^{\prime}, \mu^{\prime}\right),\left(M^{\prime \prime}, \mu^{\prime \prime}\right)$ are the outputs of Algorithm M-Basis for inputs $(G, d, \delta),(G, d / 2, \delta)$ and $\left(x^{-d / 2} M^{\prime} G, d / 2, \mu^{\prime}\right)$ respectively, then $(M, \mu)=\left(M^{\prime \prime} M^{\prime}, \mu^{\prime \prime}\right)$.

Algorithm PM-Basis $(G, d, \delta)$

Input: $G \in \mathrm{K}[[x]]^{m \times n}$ with $m \geq n, d \in \mathbb{N}$ and $\delta \in \mathbb{N}^{m}$.

Output: a $\sigma$-basis $M \in \mathrm{K}[x]^{m \times m}$ with $\sigma=n d, \mu \in \mathbb{N}^{m}$.

Condition: $d=0$ or $\log d \in \mathbb{N}$. 
if $d=0$ then $(M, \mu):=\left(I_{m}, \delta\right)$;

else if $d=1$ then $(M, \mu):=\mathrm{M}-\operatorname{Basis}(G, d, \delta)$;

else if $d \geq 2$ then

$\left(M^{\prime}, \mu^{\prime}\right):=$ PM-Basis $(G, d / 2, \delta)$;

$G^{\prime}:=x^{-d / 2} M^{\prime} G \bmod x^{d / 2}$;

$\left(M^{\prime \prime}, \mu^{\prime \prime}\right):=$ PM-Basis $\left(G^{\prime}, d / 2, \mu^{\prime}\right)$;

$(M, \mu):=\left(M^{\prime \prime} M^{\prime}, \mu^{\prime \prime}\right)$

fi;

return $(M, \mu)$;

TheOrem 2.4. Algorithm PM-Basis is correct. Its cost is $O\left(\mathrm{MM}^{\prime}(m, d)\right)$ or $O^{\sim}\left(m^{\omega} d\right)$ operations in $\mathrm{K}$.

Proof. For correctness it suffices to show that the algorithm with input $(G, d, \delta)$ uses only the first $d$ coefficients of series $G$ : when $d=1$ this is true because of Algorithm M-Basis; if we assume this is true for a given $d / 2$ then this is still true for $d$ since $x^{-d / 2} M^{\prime} G \bmod x^{d / 2}$ depends only on $G$ $\bmod x^{d}$. Correctness then follows immediately from Lemma 2.3 .

Now about complexity. First, it follows from Algorithm M-Basis that $\operatorname{deg} M \leq d$. Hence the product $M^{\prime \prime} M^{\prime}$ costs $\operatorname{MM}(m, d / 2)$. Second, since $\operatorname{deg} M^{\prime} \leq d / 2$, the coefficient in $x^{i}$ of $x^{-d / 2} M^{\prime} G \bmod x^{d / 2}$ is the coefficient in $x^{i+d / 2}$ of $M^{\prime}\left(G \bmod x^{d}\right)$. This product costs $\operatorname{MM}(m, d)$. The cost $C(m, n, d)$ of Algorithm PM-Basis thus satisfies $C(m, n, 1)=$ $O(\operatorname{MM}(m))$ and, for $d \geq 2$,

$$
C(m, n, d) \leq 2 C(m, n, d / 2)+\operatorname{MM}(m, d / 2)+\operatorname{MM}(m, d) .
$$

This gives the bound $O\left(\mathrm{MM}^{\prime}(m, d)\right)$.

\section{COLUMN REDUCTION}

For $A \in \mathrm{K}[x]^{n \times n}$ we consider the problem of computing $C \in \mathrm{K}[x]^{n \times n}$ such that $C=A U$ is column reduced, $U$ being a unimodular matrix over $\mathrm{K}[x]$. Column reduction is essentially lattice basis reduction for $\mathrm{K}[x]$-modules. To define the reduction, let $d_{j}$ denote the degree of the $j$ th column of $C$. The corresponding coefficient vector of $x^{d_{j}}$ is the $j$ th leading vector of $C$. We let $[C]_{l}$ be the matrix of these leading vectors.

Definition 3.1. A matrix $C$ is column reduced if its leading coefficient matrix satisfies rank $[C]_{l}=\operatorname{rank} C$.

We refer to $[14,23]$ and the references therein for discussions on previous reduction algorithms and applications of the form especially in linear algebra and in linear control theory. If $r$ is the rank of $A$, the best previously known cost for reducing $A$ was $O\left(n^{2} r d^{2}\right)$ operations in $\mathrm{K}$ [14]. Thus in particular $O\left(n^{3} d^{2}\right)$ for a nonsingular matrix. Here we propose a different approach which takes advantage of fast polynomial matrix multiplication and gives in particular the complexity estimate $O^{\sim}\left(n^{\omega} d\right)$.

We assume that $A$ of degree $d$ is nonsingular in $\mathrm{K}[x]^{n \times n}$ The general case would require further developments. We compute a column reduced form of $A$ by combining our techniques in [23] to the high-order lifting and the integrality certificate in [17]. The main idea is to reduce the problem to the computation of a matrix Padé approximant whose side-effect is to normalize the involved matrices [23]. Let us first recall the definition of right matrix greatest common divisors.
Definition 3.2. A right matrix gcd of $P \in \mathrm{K}[x]^{m \times n}$ and $A \in \mathrm{K}[x]^{n \times n}$ is any full row rank matrix $G$ such that

$$
U\left[\begin{array}{l}
P \\
A
\end{array}\right]=\left[\begin{array}{c}
G \\
0
\end{array}\right]
$$

with $U$ unimodular.

Right gcd's are not unique, but if $\left[\begin{array}{ll}P^{T} & A^{T}\end{array}\right]^{T}$ has full column rank - here this is true by assumption - then, for given matrices $P$ and $A$, all the gcd's are nonsingular and left equivalent (up to multiplication by a unimodular matrix on the left) in $\mathrm{K}[x]^{n \times n}$. This also leads to the notion of an irreducible matrix fraction description. (See for example [11] for a detailed study of matrix gcd's and fractions.)

Definition 3.3. If a right gcd of $P$ and $A$ is unimodular then we say that $P$ and $A$ are relatively prime and that $P A^{-1}$ is an irreducible right matrix fraction description.

The whole algorithm for column reduction will be given in Section 3.3. A matrix fraction $H \in \mathrm{K}(x)^{n \times n}$ is said to be strictly proper if it tends to zero when $x$ tends to infinity $[11, \S 6.3 .2]$. The first step of the algorithm, detailed in Section 3.1, is to compute from $A$ a strictly proper and irreducible right fraction description

$$
H=R A^{-1} \in \mathrm{K}(x)^{n \times n}, R \in \mathrm{K}[x]^{n \times n} .
$$

The fact that $H$ is strictly proper implies that the degree of the $j$ th column of $R$ must be strictly lower than the degree of the $j$ th column of $A$. Since the degrees of $R$ and $A$ are bounded by $d$, the second step of the method, studied in Section 3.2 , is to compute from the first $2 d+1$ terms of the expansion of $H$ a right matrix Padé approximant

$$
H=T C^{-1}
$$

of $H$. Such an approximant, obtained from the results of Section 2 and [2], will have the additional property that $C$ is column reduced. We will see that by the equivalence of irreducible fractions, $C$ will be a column reduced form of $A$.

Like the algorithms in [17], our column reduction algorithm is randomized Las Vegas since the first step requires that $\operatorname{det} A(0) \neq 0$. Without loss of generality this may be assumed by choosing a random element $x_{0}$ in $\mathrm{K}$ and by computing a column reduced form $C$ of $A\left(x+x_{0}\right)$. Indeed, a column reduced form of $A$ is then recovered as $C\left(x-x_{0}\right)$.

\subsection{A Strictly Proper and Irreducible Fraction}

For a given $A$, its inverse $A^{-1}$ may not be a strictly proper rational function, a case where $R=I$ is not a suitable choice in (6). We show that the integrality certificate of $[17, \S 11]$ can be used here to find a target strictly proper function.

Lemma 3.4. Let $A \in \mathrm{K}[x]^{n \times n}$ of degree $d$ be such that $\operatorname{det} A(0) \neq 0$. For $h>(n-1) d$ define $R \in \mathrm{K}[x]^{m \times n}$ by

$$
I=\left(A^{-1} \bmod x^{h}\right) A+x^{h} R .
$$

The fraction $R A^{-1}$ is strictly proper and irreducible. If $h$ is the closest power of 2 greater than $(n-1) d+1$, the $2 d+1$ first terms of the expansion of $R A^{-1}$ may be computed at the cost of $O\left(\mathrm{MM}(n, d) \log n+\mathrm{MM}^{\prime \prime}(n, d)\right)+O^{\sim}\left(n^{2} d\right)$ operations in $\mathrm{K}$. 
Proof. Identity (7) is identity (18) in [17] with $B=I$ and $T=A$. This is a Euclidean matrix division with coefficients in reverse order. The fraction $R A^{-1}$ is strictly proper because

$$
R A^{-1}=x^{-h} A^{-1}-x^{-h}\left(A^{-1} \bmod x^{h}\right)
$$

and $h>(n-1) d \geq \operatorname{deg} A^{*}$ where $A^{*}$ is the adjoint matrix of $A$. On the other hand, there is a unimodular $U$ such that

$$
\left[\begin{array}{c}
x^{h} R \\
A
\end{array}\right]=\left[\begin{array}{cc}
I & -\left(A^{-1} \bmod x^{h}\right) \\
0 & I
\end{array}\right]\left[\begin{array}{l}
I \\
A
\end{array}\right]=U\left[\begin{array}{l}
I \\
0
\end{array}\right] .
$$

Hence matrices $x^{h} R$ and $A$ are relatively prime (see Definition 3.2) and the same is true for $R$ and $A$.

For $h$ as in the statement, the $2 d+1$ terms of the expansion of $R A^{-1}$ may be computed by high-order $x^{d}$-lifting $[17, \S 10]$ with input parameters $A, I, h / d$ and 3 . The corresponding cost is given by [17, Proposition 17].

\subsection{Padé Approximation and Reduction}

The key observation is that the descriptions $T C^{-1}$ of $H$ with $C$ a column reduced form of $A$ are those whose numerator and denominator matrices have minimal degrees (see Corollary 3.6 below). By definition they satisfy

$$
\left[\begin{array}{ll}
H & -I
\end{array}\right]\left[\begin{array}{l}
C \\
T
\end{array}\right]=0 \bmod x^{2 d+1}
$$

and, as we shall see, their minimality implies that they must appear in any $\sigma$-basis of $G=[H-I]$ for $\sigma=n(2 d+1)$. (Here we consider $\sigma$-bases with respect to the columns rather than the rows. Hence we transpose the matrices of Section 2.)

To describe the set of all matrices $T$ and $C$ we use the notion of minimal basis of a module. For $M \in \mathrm{K}(x)^{n \times m}$, $m>n$, with rank $n$, let $N \in \mathrm{K}[x]^{m \times(m-n)}$ with columns forming a basis of the $\mathrm{K}[x]$-submodule ker $M$. We denote by $d_{1}, d_{2}, \ldots, d_{m-n}$ the column degrees of $N$ and assume they are ordered as $d_{1} \leq d_{2} \leq \cdots \leq d_{m-n}$. Then we have the following theorem and consequence.

THEOREM 3.5. [11, §6.5.4]. If $N$ is column reduced then the column degrees $d_{1}^{\prime} \leq d_{2}^{\prime} \leq \cdots \leq d_{m-n}^{\prime}$ of any other basis of $\operatorname{ker} M$ satisfy $d_{j}^{\prime} \geq \bar{d}_{j}$ for $1 \leq j \leq m-n$. We say that the columns of $N$ form a minimal basis of $\operatorname{ker} M$.

Corollary 3.6. A basis $\left[C^{T} T^{T}\right]^{T}$ of $\operatorname{ker} G=\operatorname{ker}[H-I]$ is minimal if and only if $C$ is a column reduced form of $A$.

Proof. If $\left[C^{T} T^{T}\right]^{T}$ is a minimal basis then $H=T C^{-1}$ must be irreducible, otherwise the simplification of $(T, C)$ by a right matrix gcd would lead to a basis with smaller degrees. The latter would contradict Theorem 3.5. In addition since $\left[C^{T} T^{T}\right]^{T}$ is column reduced then $C$ is column reduced. Indeed, $H$ being strictly proper implies that $T$ has column degrees strictly lower that those of $C$ which thus dominate. By [11, Theorem 6.5-4] we further know that two irreducible descriptions $T C^{-1}$ and $R A^{-1}$ of the same function $H$ have equivalent denominators. This means that there exists a unimodular $U$ such that $C=A U$. Hence $C$ is a column reduced form of $A$. Conversely, if $C$ in a basis $\left[C^{T} T^{T}\right]^{T}$ is a column reduced form of $A$ then by Theorem 6.5-4 cited above, $T C^{-1}$ is an irreducible description of $H$. Since $C$ is column reduced, the non-minimality of $\left[C^{T} T^{T}\right]^{T}$ as a basis of ker $G$ would then contradict its irreducibility.
We now show that, for $\sigma$ large enough, a $\sigma$-basis with respect to the columns of $\left[\begin{array}{ll}H & -I\end{array}\right]$ leads to a minimal basis $\left[\begin{array}{ll}C^{T} & T^{T}\end{array}\right]^{T}$ as in the corollary, and hence to a column reduced form of $A$. We follow here the techniques in [10] for computing a minimal basis of the kernel of a polynomial matrix.

Lemma 3.7. Let $N \in \mathrm{K}[x]^{2 n \times 2 n}$ be a $\sigma$-basis with respect to the columns of $G=[H-I]$. If $\sigma \geq n(2 d+1)$, then the $n$ columns of $N$ of degree at most d define an irreducible description $T C^{-1}$ of $H$ with $C$ a column reduced form of $A$.

Proof. We first show that there may be at most one set of $n$ columns of $N$ of degree at most $d$. Then the minimality of the $\sigma$-basis will imply its existence and the fact that it leads to a fraction description of the form $T C^{-1}$.

If $\left[Q^{T} P^{T}\right]^{T}$ is a set of $n$ columns of $N$ of degrees bounded by $d$ then

$$
H Q-P \equiv 0 \bmod x^{2 d+1} .
$$

If $A^{-1} S$ is a left description of $H$, defined in the same way as $R A^{-1}$ in Lemma 3.4 , we get

$$
S Q-A P \equiv 0 \bmod x^{2 d+1} .
$$

Since every matrix in the latter identity has degree at most $d$ we deduce that

$$
S Q-A P=0 .
$$

It follows from the columns of a $\sigma$-basis $N$ being linearly independent over $\mathrm{K}(x)[2]$ that $\left[Q^{T} P^{T}\right]^{T}$ has full column rank. Since (9) implies that

$$
\left[\begin{array}{cc}
I & 0 \\
S & -A
\end{array}\right]\left[\begin{array}{l}
Q \\
P
\end{array}\right]=\left[\begin{array}{l}
Q \\
0
\end{array}\right]
$$

we see that $Q$ is invertible and satisfies

$$
P Q^{-1}=A^{-1} S=H \text {. }
$$

Another choice $\left[\begin{array}{ll}Q_{1}^{T} & P_{1}^{T}\end{array}\right]^{T}$ of $n$ such columns would give $H=P Q^{-1}=P_{1} Q_{1}^{-1}$. By $[11$, Theorem 6.5-4] the two descriptions would verify

$$
\left[\begin{array}{l}
Q \\
P
\end{array}\right] U=\left[\begin{array}{l}
Q_{1} \\
P_{1}
\end{array}\right] \text { with } U \text { unimodular, }
$$

and this would contradict the nonsingularity of the $\sigma$-basis. Hence the choice $\left[\begin{array}{ll}Q^{T} & P^{T}\end{array}\right]^{T}$ must be unique as announced.

Let $d_{1}, \ldots, d_{n}$ be the minimal degrees among the columns of a minimal description $\left[\begin{array}{cc}C^{T} & T^{T}\end{array}\right]^{T}$ and let $v_{1}, \ldots, v_{n}$ be the corresponding columns. From II) in Definition 2.1, $v_{1}$ can be written as

$$
v_{1}=\sum_{j=1}^{2 n} c_{1}^{(j)} N_{j} \quad \text { with } \operatorname{deg} c_{1}^{(j)}+\operatorname{deg} N_{j} \leq d_{1}
$$

and where $N_{j}$ is the $j$ th column of the $\sigma$-basis $N$. Thus one column of $N$ has degree bounded by $d_{1}$. Now assume that $N$ has $k-1$ columns of degrees $d_{1}, \ldots, d_{k-1}$ with $v_{k}$ not belonging to the corresponding submodule. As for $k=$ 1 , there exists a column of $N$, linearly independent with respect to the first $k-1$ chosen ones, of degree bounded by $d_{k}$. Therefore $N$ contains $n$ distinct columns of degrees bounded by $d_{1}, \ldots d_{n}$ and, by (10), in the kernel of $[H-I]$. Lemma 3.7 shows in conclusion that these $n$ columns give $C$, a column reduced form of $A$, in their first $n$ rows.

We may notice that the result of the lemma would be true as soon as $\sigma>2 n d$ for the computation of an approximant of type $(d-1, d)$ as defined in [2]. 


\subsection{Cost of the Reduction}

Our column reduction algorithm can be stated as follows.

Algorithm ColumnReduction $(A)$

Input: $A \in \mathrm{K}[x]^{n \times n}$ of degree $d$.

Output: $C=A U$ a column reduced form of $A$.

Condition: $A$ is nonsingular.

Choice of a random $x_{0}$ in $\mathrm{K}$;

if $\operatorname{det} A\left(x_{0}\right)=0$ then fail; /* $A$ is probably singular*/

$B:=A\left(x+x_{0}\right)$;

$h:=(n-1) d+1$;

$H:=\left(B^{-1}-\left(B^{-1} \bmod x^{h}\right)\right) / x^{h} \bmod x^{2 d+1}$;

$T C^{-1}:=$ a Padé approximant of $H \bmod x^{2 d+1}$;

return $C\left(x-x_{0}\right)$;

Its complexity follows from Lemma 3.4 concerning the computation of the first terms of $H$, and from Theorem 2.4 concerning the computation of the $n(2 d+1)$-basis of Lemma 3.7.

THEOREM 3.8. A column reduced form of a nonsingular matrix $A$ of degree $d$ in $\mathrm{K}[x]^{n \times n}$ can be computed by a Las Vegas (certified) algorithm in $O\left(\operatorname{MM}(n, d) \log n+\mathrm{MM}^{\prime}(n, d)+\right.$ $\left.\mathrm{MM}^{\prime \prime}(n, d)\right)+O^{\sim}\left(n^{2} d\right)$ or $O^{\sim}\left(n^{\omega} d\right)$ operations in $\mathrm{K}$.

\section{MATRIX PRODUCT \& DETERMINANT}

The link between matrix multiplication and determinant computation over a field $\mathrm{K}$ is well known. We may refer to [5, Chap.16] for a survey of the question. If we have an algorithm for multiplying two matrices with $\operatorname{MM}(n)$ operations in $\mathrm{K}$ then we have an algorithm (algebraic RAM) for computing the determinant with $O(\operatorname{MM}(n))$ operations in $\mathrm{K}$ [4]. Conversely, the exponents (computation trees) of matrix multiplication and of determinant computation coincide $[19,1]$. Furthermore, if we have a randomized Monte Carlo algorithm which computes the determinant with $D(n)$ operations in $\mathrm{K}$ then we have a Monte Carlo algorithm for multiplying two matrices with $O(D(n))$ operations in $\mathrm{K}[8$, Theorem 1.3].

In this section we show that similar results hold for polynomial matrices of degree $d$. In Section 4.1, using a slight extension of Baur and Strassen's idea [1, Corollary 5], we propose a reduction of polynomial matrix multiplication to determinant computation. Then in Section 4.2, based on the techniques in $[16,17,22,10]$, we investigate the reverse reduction.

We use two models of computation, algebraic straight-line programs or algorithms on an algebraic RAM.

\subsection{Polynomial Matrix Multiplication}

Baur and Strassen [1, Corollary 5] in conjunction with [19, 4] have shown that a straight-line program of length $D(n)$ for computing the determinant of a matrix $A$ in $\mathrm{K}^{n \times n}$ can be transformed into a program of length bounded by $O(D(n))$ for matrix multiplication. Indeed, the problem of multiplying two matrices can be reduced to matrix inversion [19, 4]. Then matrix inversion is reduced to the problem of computing the determinant by differentiation of the program of length $D(n)[12,1]$.

The complexity estimate $O(D(n))$ for matrix multiplication relies on the computation of the partial derivatives of the determinant as a function in $\mathrm{K}\left[a_{1,1}, \ldots, a_{i, j}, \ldots, a_{n, n}\right]$.
The $a_{i, j}$ 's are indeterminates standing for the entries of the input matrix. It was not clear how to extend the result to polynomial matrices. The output of a program of length $D(n, d)$ over $\mathrm{K}$ which computes the determinant of a polynomial matrix is a function in $\mathrm{K}\left[x, a_{1,1}, \ldots, a_{i, j}, \ldots, a_{n, n}\right]$, that is, a set of functions in $\mathrm{K}\left[a_{1,1}, \ldots, a_{i, j}, \ldots, a_{n, n}\right]$. $\mathrm{A}$ straightforward idea could be to differentiate at least $d$ such functions, but it is not known how to do it without increasing the complexity estimate $O(D(n, d))$.

Here we remark that having only one particular coefficient of the polynomial matrix determinant is sufficient for recovering the first $d+1$ coefficients of the polynomial entries of the adjoint matrix $A^{*}=(\operatorname{det} A) A^{-1} \in \mathrm{K}[x]^{n \times n}$. Hence we first compute $A^{*}$ modulo $x^{d+1}$ and from there, the multiplication of two matrices of degree $d$ is easily deduced.

Let $A \in \mathrm{K}[x]^{n \times n}$ have degree $d$ and denote its $(i, j)$ entry by $a_{i, j}=\sum_{k=0}^{d} a_{i, j, k} x^{k}$. Let further $a_{i, j}^{*}=\sum_{k=0}^{n d-d} a_{i, j, k}^{*} x^{k}$ be the $(i, j)$ entry of the adjoint matrix $A^{*}$ of $A$ and let $\Delta=$ $\sum_{l=0}^{n d} \Delta_{l} x^{l}$ be the determinant of $A$. We have the following relation between the partial derivatives of coefficient $\Delta_{l}$ and some of the $a_{i, j, k}^{*}$ 's.

LEMmA 4.1. The partial derivatives of the coefficients of the determinant and the coefficients of the adjoint matrix satisfy

$$
a_{j, i, l-k}^{*}=\frac{\partial \Delta_{l}}{\partial a_{i, j, k}}, 0 \leq l \leq n d, 0 \leq k \leq d .
$$

where, by convention, $a_{j, i, k}^{*}=0$ if $k<0$ or $k>n d-d$.

Proof. By Cramer's rule and since $\partial a_{i, j} / \partial a_{i, j, k}=x^{k}$, we have $\partial \Delta / \partial a_{i, j, k}=x^{k} a_{j, i}^{*}$. On the other hand, for $1 \leq k \leq$ $d$ the coefficients $\Delta_{0}, \ldots, \Delta_{k-1}$ do not depend on variable $a_{i, j, k}$ and thus $\partial \Delta / \partial a_{i, j, k}=\sum_{l=k}^{n d}\left(\partial \Delta_{l} / \partial a_{i, j, k}\right) x^{l}$. Therefore

$$
\sum_{l=0}^{n d-d} a_{j, i, l}^{*} x^{k+l}=\sum_{l=0}^{n d-k} \frac{\partial \Delta_{k+l}}{\partial a_{i, j, k}} x^{k+l}
$$

and the result follows by identifying the coefficients.

The theorem below is given for programs over $\mathrm{K}$ which compute the particular coefficient $\Delta_{d}$. It thus remains valid for programs over $\mathrm{K}$ which compute the whole determinant in $\mathrm{K}[x]$.

THEOREM 4.2. If there is a straight-line program of length $D(n, d)$ over $\mathrm{K}$ which computes the $(d+1)$ st coefficient of the determinant of an $n \times n$ matrix of degree $d$, then there is a straight-line program of length no more than $8 D(n, d)$ which multiplies two $n \times n$ matrices of degree $d$.

Proof. It follows from Lemma 4.1 with $l=d$ that the first $d+1$ coefficients of $a_{j, i}^{*}$ are given by

$$
a_{j, i, d-k}^{*}=\frac{\partial \Delta_{d}}{\partial a_{i, j, k}}, \quad 0 \leq k \leq d .
$$

By computing the partial derivatives $[12,1]$ of the given program for the determinant coefficient $\Delta_{d}$ we thus have a program of length bounded by $4 D(n, d)$ for computing $A^{*}$ $\bmod x^{d+1}$. We conclude by applying this result twice to the well known $3 n \times 3 n$ matrix

$$
A=\left[\begin{array}{ccc}
I_{n} & A_{1} & \\
& I_{n} & A_{2} \\
& & I_{n}
\end{array}\right] \text { with } A_{1}, A_{2} \in \mathrm{K}[x]^{n \times n} \text { of degree } d .
$$


The associated adjoint matrix is the matrix of degree $2 d$

$$
A^{*}=\left[\begin{array}{ccc}
I_{n} & -A_{1} & A_{1} A_{2} \\
& I_{n} & -A_{2} \\
& & I_{n}
\end{array}\right] .
$$

One can thus recover $A_{1} A_{2} \bmod x^{d+1}$ from $A^{*} \bmod x^{d+1}$. To get higher order terms, notice that if $A_{1} A_{2}=x^{d} H+L$ then $\bar{H}=\bar{A}_{1} \bar{A}_{2} \bmod x^{d+1}$ where $\bar{M}=\sum_{i=0}^{d} M_{d-i} x^{i}$ is the reciprocal matrix polynomial of $M=\sum_{i=0}^{d} M_{i} x^{i}$. Therefore $\bar{H}$ and thus $H$ can be recovered from $\bar{A}^{*} \bmod x^{d+1}$.

Following Giesbrecht [8, Theorem 1.3] we may state an analogous result for algorithms on an algebraic RAM: if we have a randomized Monte Carlo algorithm which computes $\Delta_{d}$ with $D(n, d)$ operations in $\mathrm{K}$ then we have a Monte Carlo algorithm for multiplying two matrices of degree $d$ with $O(D(n, d))$ operations in $\mathrm{K}$.

\subsection{Polynomial Matrix Determinant}

Over K, algorithms for reducing determinant computation to matrix multiplication work recursively in $O(\log n)$ steps. Roughly, step $i$ involves $n / 2^{i}$ products of $2^{i} \times 2^{i}$ matrices. (See for example $[18,4]$.) When looking for the determinant of an $n \times n$ polynomial matrix of degree $d$, both Storjohann's algorithm $[16,17]$ and the straight-line program we derive below from our previous studies in [22, 10], also work in $O(\log n)$ steps. They involve polynomial matrices of dimensions $2^{i} \times 2^{i}$ and degree $n d / 2^{i}$ (this accounts for the definition of function $\mathrm{MM}^{\prime \prime}(n, d)$ in introduction).

In this section we study the costs of these two different methods for the polynomial matrix determinant. The first one is Storjohann's high order lifting on an algebraic RAM $[16,17]$. We recall the result briefly in Section 4.2 .1 for the sake of completeness. We then present in Section 4.2.2 an alternative approach for straight-line programs, which has been developed independently $[22,10]$. The complexity estimates are given in terms of $\operatorname{MM}^{\prime}(n, d)$ and $\operatorname{MM}^{\prime \prime}(n, d)$ and they all reduce to $O^{\sim}\left(n^{\omega} d\right)$ when taking, for instance, $\operatorname{MM}(n, d)=\Theta\left(n^{\omega} d \log d \log \log d\right)$.

\subsubsection{Lifting Determinant Algorithms}

Storjohann gives in [17, Proposition 41] a Las Vegas algorithm for computing the determinant of a polynomial matrix in $O^{\sim}\left(n^{\omega} d\right)$ operations.

TheOREM 4.3. [17] The determinant of an $n \times n$ polynomial matrix of degree $d$ can be computed by a Las Vegas algorithm in $O\left(\mathrm{MM}(n, d) \log ^{2} n+\sum_{i=0}^{\log n} 2^{i} \mathrm{MM}^{\prime \prime}\left(2^{-i} n, 2^{i} d\right)\right)+$ $O^{\sim}\left(n^{2} d\right)$ or $O^{\sim}\left(n^{\omega} d\right)$ operations in $\mathrm{K}$.

The term involving $\mathrm{MM}^{\prime \prime}$ comes from the integrality certificate and the Smith form computations of [17, Propositions $21 \& 40]$. The term in $O\left(\operatorname{MM}(n, d) \log ^{2} n\right)$ comes from the high-order lifting of [17, Prop. 17] performed at each step of the $O(\log n)$ steps of the main iteration $[17, \S 17]$.

\subsubsection{Straight-line Determinant}

Given $A \in \mathrm{K}[x]^{n \times n}$ of degree $d$ and sufficiently generic, the straight-line approach presented in $[22,10]$ computes the inverse of $A$ as $A^{-1}=B^{-1} U$ where $U \in \mathrm{K}[x]^{n \times n}$ and $B \in \mathrm{K}[x]^{n \times n}$ is diagonal of degree $n d$. One can further recover the determinant of $A$ from $B$ alone as explained below. By definition of the inverse, $U=(\operatorname{det} A)^{-1} B A^{*}$ where
$A^{*}$ is the adjoint matrix of $A$. Generically, $\operatorname{deg} \operatorname{det} A=$ $\operatorname{deg} B=n d$ and $\operatorname{det} A$ is coprime with each entry $a_{i, j}^{*}$ of $A^{*}$. It follows that the diagonal entries $b_{i, i}$ of $B$ are nonzero constant multiples of $\operatorname{det} A$. Since $\operatorname{det} A(0)$ is generically nonzero, the determinant of a generic $A$ is thus equal to $(\operatorname{det} A(0)) b_{i, i} / b_{i, i}(0)$ for $1 \leq i \leq n$.

In [10], Algorithm Inverse computes $B$ by diagonalizing the input matrix in $\log n$ steps of the form

$$
A \rightarrow U A=\left[\begin{array}{l}
\bar{U} \\
\underline{U}
\end{array}\right]\left[\begin{array}{ll}
A_{L} & A_{R}
\end{array}\right]=\left[\begin{array}{ll}
\bar{U} A_{L} & \\
& \underline{U} A_{R}
\end{array}\right]
$$

where $A_{L}, A_{R} \in \mathrm{K}[x]^{n \times n / 2}$ and where $\bar{U}, \underline{U} \in \mathrm{K}[x]^{n / 2 \times n}$ are minimal bases of the left kernels of $A_{R}, \bar{A}_{L}$ respectively. These minimal bases are as in Theorem 3.5, for left kernels.

Now for computing $\operatorname{det} A$ we only need the $(1,1)$ entry of $B$ and $\log n$ "compression" steps of the form

$$
A \rightarrow \bar{U} A_{L}
$$

thus suffice. Hence the algorithm below, kindly suggested by one of the referees.

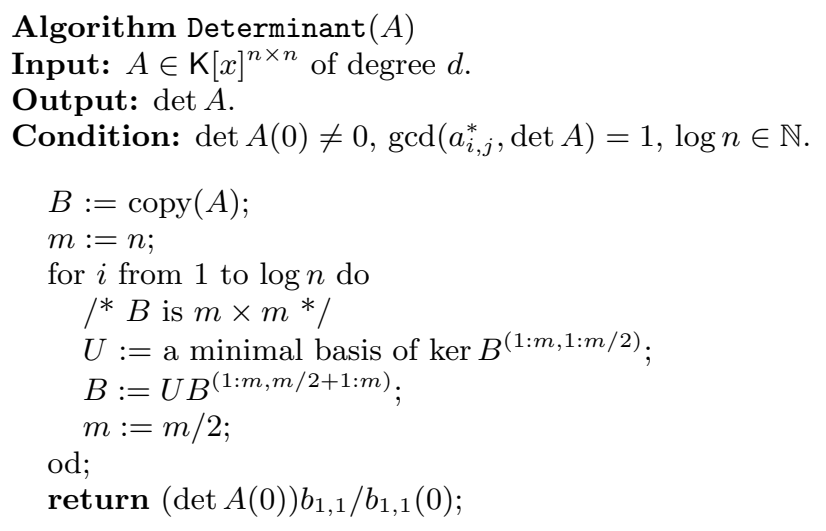

The analysis of this algorithm is similar to the one of the inversion algorithm in [10] and we simply recall the key point for complexity: although the minimal bases in (11) can have degrees as large as $n d$, they generically have degrees equal to $d$ - a property which carries over the next step - and can then be recovered from the rows of any $\sigma$-bases of $A_{L}, A_{R}$ with $\sigma \geq n(2 d+1) \quad[10$, Properties 1 and 2]. Step $i$ in Algorithm Determinant thus generically computes a $B$ of dimensions $2^{-i} n \times 2^{-i} n$ and degree $2^{i} d$; it then follows from Theorem 2.4 that at step $i+1$ the minimal basis $U$ and the update of $B$ can both be computed in $O\left(\operatorname{MM}^{\prime}\left(2^{-i} n, 2^{i} d\right)\right)$ operations in $\mathrm{K}$.

When $n$ is not a power of two, one may augment $A$ as

$$
\mathcal{A}=\left[\begin{array}{cc}
A & \\
X & I_{2^{p}-n}
\end{array}\right] \text { where } 2^{p-1}<n<2^{p} .
$$

When both $A$ and $X$ are generic polynomial matrices of degree $d$, the $p$ minimal bases in $\operatorname{Determinant}(\mathcal{A})$ will have degrees bounded by $d, 2 d, 4 d, \ldots, 2^{p-1} d$ and the asymptotic complexity of computing these bases remains the same as in the previous paragraph.

Recalling that the cost of computing $\operatorname{det} A(0)$ is bounded by $O(\operatorname{MM}(n))$, we thus have the following for any positive integer $n$.

THEOREM 4.4. The determinant of an $n \times n$ polynomial matrix of degree $d$ can be computed by a straight-line program over $\mathrm{K}$ of length $O\left(\sum_{i=0}^{\log n} \mathrm{MM}^{\prime}\left(2^{-i} n, 2^{i} d\right)\right)$ or $O^{\sim}\left(n^{\omega} d\right)$. 


\section{CONCLUSION}

In this paper we reduced polynomial matrix multiplication to determinant computation and conversely, under the straight-line model. Under the algebraic RAM model, we reduced the tasks of computing a $\sigma$-basis and column reduced form to the one of multiplying square polynomial matrices; as we have seen, similar reductions follow from [17] for the problems of computing the determinant and the Smith normal form.

However, in $\mathrm{K}[x]^{n \times n}$ it is still unclear whether

- Hermite and Frobenius normal forms,

- associated transformation matrices (even for the column reduced form),

- the characteristic polynomial

can be computed in $O^{\sim}(\operatorname{MM}(n, d))$ or $O^{\sim}\left(n^{\omega} d\right)$ operations in $\mathrm{K}$ as well. Another related question is whether the straightline approach of Section 4.2.2 yields a $O^{\sim}\left(n^{3} d\right)$ algorithm for computing the inverse of a polynomial matrix.

\section{REFERENCES}

[1] W. Baur and V. Strassen. The complexity of partial derivatives. Theoretical Computer Science, 22:317-330, 1983.

[2] B. Beckermann and G. Labahn. A uniform approach for the fast computation of matrix-type Padé approximants. SIAM Journal on Matrix Analysis and Applications, 15(3):804-823, 1994.

[3] D. Bini and V.Y. Pan. Polynomial and Matrix Computations, Vol 1: Fundamental Algorithms. Birkhauser, Boston, 1994.

[4] J. Bunch and J. Hopcroft. Triangular factorization and inversion by fast matrix multiplication. Mathematics of Computation, 28:231-236, 1974.

[5] P. Bürgisser, M. Clausen, and M.A. Shokrollahi. Algebraic Complexity Theory, volume 315 of Grundlehren der mathematischen Wissenschaften. Springer-Verlag, 1997.

[6] D.G. Cantor and E. Kaltofen. On fast multiplication of polynomials over arbitrary algebras. Acta Informatica, 28(7):693-701, 1991.

[7] D. Coppersmith. Solving homogeneous linear equations over $\mathrm{GF}(2)$ via block Wiedemann algorithm. Mathematics of Computation, 62(205):333-350, 1994.

[8] M. Giesbrecht. Nearly optimal algorithms for canonical matrix forms. PhD thesis, Department of Computer Science, University of Toronto, 1993.

[9] O.H. Ibarra, S. Moran, and R. Hui. A generalization of the fast LUP matrix decomposition algorithm and applications. Journal of Algorithms, 3:45-56, 1982.

[10] C.-P. Jeannerod and G. Villard. Straight-line computation of the polynomial matrix inverse. Research Report 2002-47, Laboratoire LIP, ENS Lyon, France. http://www . ens-lyon.fr/LIP/Pub/rr2002.html.
[11] T. Kailath. Linear systems. Prentice Hall, 1980.

[12] S. Linnainmaa. Taylor expansion of the accumulated rounding errors. BIT, 16:146-160, 1976.

[13] A. Lobo. Matrix-free linear system solving and applications to symbolic computation. $\mathrm{PhD}$ thesis, Dept. Comp. Sc., Rensselaer Polytech. Instit., Troy, New York, Dec. 1995.

[14] T. Mulders and A. Storjohann. On lattice reduction for polynomial matrices. Journal of Symbolic Computation, 35(4):377-401, 2003.

[15] A. Storjohann. Algorithms for Matrix Canonical Forms. PhD thesis, Institut für Wissenschaftliches Rechnen, ETH-Zentrum, Zurich, Switzerland, November 2000.

[16] A. Storjohann. High-order lifting (extended abstract). In Internat. Symp. Symbolic Algebraic Comput., Lille, France, pages 246-254. ACM Press, July 2002.

[17] A. Storjohann. High-order lifting and integrality certification. Journal of Symbolic Computation, special issue on papers of the 2002 Internat. Symp. Symbolic Algebraic Comput., M. Giusti and L.M. Pardo, editors, 2003. To appear, 44 pages.

[18] V. Strassen. Gaussian elimination is not optimal. Numerische Mathematik, 13:354-356, 1969.

[19] V. Strassen. Vermeidung von Divisionen. J. Reine Angew. Math., 264:182-202, 1973.

[20] E. Thomé. Subquadratic computation of vector generating polynomials and improvement of the block Wiedemann algorithm. Journal of Symbolic Computation, special issue on papers of the 2001 Internat. Symp. Symbolic Algebraic Comput., 33(5):757-775, 2002.

[21] W.J. Turner. Black box linear algebra with the LinBox library. PhD thesis, North Carolina State University, Raleigh, NC USA, May 2002.

[22] G. Villard. Computation of the inverse and determinant of a matrix. Algorithms Seminar 2001 2002, F. Chyzak, editor. INRIA Rocquencourt, France, 2003.

[23] G. Villard. Computing Popov and Hermite forms of polynomial matrices. In Internat. Symp. Symbolic Algebraic Comput., Zurich, Suisse, pages 250-258. ACM Press, July 1996.

[24] G. Villard. Further analysis of Coppersmith's block Wiedemann algorithm for the solution of sparse linear systems. In Internat. Symp. Symbolic Algebraic Comput., Maui, Hawaii, USA, pages 32-39. ACM Press, July 1997. 